\title{
Theorems of relations between elastic modulus and the stiffness matrix coefficients of isotropic homogeneous finite elements
}

\author{
Aleksandr Matveev ${ }^{1, *}$ \\ ${ }^{1}$ ICM SB RAS, 50, bil. 44, Akademgorodok, Krasnoyarsk, Russia, 660036
}

\begin{abstract}
This paper formulates theorems establishing a mutually unambiguous relation between the stiffness matrix coefficients and elastic moduli of an isotropic homogeneous finite element (FE), which allows explicitly expressing the elastic moduli of the FE via a group of its stiffness matrix coefficients.
\end{abstract}

\section{Introduction}

The calculation of elastic composite bodies of regular structure is widely performed using microand macroapproaches. According to the microapproach, the finite-element analysis of a stress state of composite bodies comes down to solving the problem of inverting high-order matrices. According to the macroapproach, a composite body is considered to be a homogeneous body with some (apparent) elastic moduli. However, determining apparent elastic moduli for threedimensional composite bodies is quite a difficult task, especially for bodies with a complex inhomogeneous structure and small filling ratio. In this paper, theorems are formulated that allow us to Express the elastic modules of FE explicitly through the group of its coefficients of the stiffness matrix, i.e. to build R-relations. The representative volume of a composite body of regular structure is a representative finite element (RFE) that comprises a finite number of regular cells with inhomogeneous structure and is considered as an isotropic homogeneous FE. The advantages of constructing apparent elastic moduli using $R$ relations are as follows. This procedure uses an arbitrarily small partition of the RFE, which can arbitrarily accurately account for a complex inhomogeneous (microinhomogeneous) structure of regular cells of the RFE within the framework of the microapproach. The proposed procedure is applied for determining apparent elastic moduli of two- or three-dimensional composite bodies of regular structure with an arbitrary filling ratio, has a matrix formulation, and is implemented on the basis of finite element method algorithms.

\section{Representation of stiffness matrix coefficients of homogeneous finite elements in explicit form via elastic moduli}

The finite element method (FEM) based calculations [1-4] of the three-dimensional stress strain state (SSS) of composite constructions of regular structure within the framework of

* Corresponding author: mtv241@mail.ru 
the macroapproach [5] come down to forming discrete models with a very high dimension. The dimensions of discrete models are reduced using multigrid finite elements (MgFE) [68]. However, the analysis of the SSS of constructions with complex microinhomogeneous structures using MgFE is very complicated. Determining apparent elastic moduli for threedimensional composite constructions is quite a difficult task, especially for bodies with a complex inhomogeneous structure and small filling ratio. Different approximate approaches to calculating the SSS of composite constructions [9-15] are applied, based on hypotheses, which generates approximate solutions with an inherent error. In this paper, it is proposed to determine apparent elastic moduli of composite bodies of regular structure using $R$ relations, which explicitly represent the elastic moduli of an isotropic homogeneous finite element (FE) via a group of its stiffness matrix coefficients. The representation of the stiffness matrix coefficients of homogeneous linear-elastic FE in explicit form via elastic moduli is shown below.

Let there be an arbitrarily shaped homogeneous FE $V_{e}$ of any order in a threedimensional problem of the elasticity theory, located in a Cartesian coordinate system $O x y z$, for which the Hooke and Cauchy expressions are fulfilled [16], i.e.,

$$
\begin{gathered}
\{\sigma\}=[D]\{\varepsilon\}, \\
\varepsilon_{x}=\frac{\partial u}{\partial x}, \varepsilon_{y}=\frac{\partial v}{\partial y}, \varepsilon_{z}=\frac{\partial w}{\partial z}, \gamma_{x y}=\frac{\partial u}{\partial y}+\frac{\partial v}{\partial x}, \gamma_{x z}=\frac{\partial u}{\partial z}+\frac{\partial w}{\partial x}, \\
\gamma_{z y}=\frac{\partial v}{\partial z}+\frac{\partial w}{\partial y},
\end{gathered}
$$

where $\{\sigma\}=\left\{\sigma_{x}, \sigma_{y}, \sigma_{z}, \tau_{y z}, \tau_{x z}, \tau_{x y}\right\}^{T}$ and $\{\varepsilon\}=\left\{\varepsilon_{x}, \varepsilon_{y}, \varepsilon_{z}, \gamma_{y z}, \gamma_{x z}, \gamma_{x y}\right\}^{T}$ are the stress and strain vectors; $u, v$, and $w$ are the displacement vectors of the element $V_{e} ;[D]$ is the matrix of the elastic moduli $C_{i j}$ of the FE $V_{e} ; C_{i j}=C_{j i}, i, j=1, \ldots, 6 ; T$ denotes transposition.

Based on the FEM, the approximating displacement functions $u, v$, and $w$ of the element $V_{e}$ are represented by the equation

$$
\left\{\begin{array}{l}
u \\
v \\
w
\end{array}\right\}=[N]\{\delta\},
$$

where $\{\delta\}=\left\{\delta_{1}, \ldots, \delta_{3 n}\right\}^{T}$ denotes the vector of nodal unknowns of the FE $V_{e}, \delta_{1}, \ldots, \delta_{n}$ denotes the nodal values of the displacements $u, \delta_{n+1}, \ldots, \delta_{2 n}$ denotes the nodal values of the displacements $v, \delta_{2 n+1}, \ldots, \delta_{3 n}$ denotes the nodal values of the displacements $w$, and $[N]$ is the shape function matrix of the form

$$
[N]=\left[\begin{array}{ccc}
N_{1}, \ldots, N_{n}, & 0, \ldots, 0, & 0, \ldots, 0 \\
0, \ldots, 0, & N_{1}, \ldots, N_{n}, & 0, \ldots, 0 \\
0, \ldots, 0, & 0, \ldots, 0, & N_{1}, \ldots, N_{n}
\end{array}\right],
$$

where $N_{\alpha}$ refers to the shape functions of the FE $V_{e}$ and $\alpha=1, \ldots, n$, where $n$ is the total number of shape functions.

The strain energy $W_{e}$ of the element $V_{e}$, used to determine the stiffness matrix of the FE $V_{e}$, is written as 


$$
\begin{gathered}
W_{e}=\varepsilon_{x}\left(\frac{1}{2} C_{11} \varepsilon_{x}+C_{12} \varepsilon_{y}+C_{13} \varepsilon_{z}+C_{14} \gamma_{y x}+C_{15} \gamma_{x z}+C_{16} \gamma_{x y}\right)+ \\
+\varepsilon_{y}\left(\frac{1}{2} C_{22} \varepsilon_{y}+C_{23} \varepsilon_{z}+C_{24} \gamma_{y x}+C_{25} \gamma_{x z}+C_{26} \gamma_{x y}\right)+ \\
+\varepsilon_{z}\left(\frac{1}{2} C_{33} \varepsilon_{z}+C_{34} \gamma_{y x}+C_{35} \gamma_{x z}+C_{36} \gamma_{x y}\right)+\gamma_{y z}\left(\frac{1}{2} C_{44} \gamma_{y x}+C_{45} \gamma_{x z}+C_{46} \gamma_{x y}\right)+ \\
+\gamma_{x z}\left(\frac{1}{2} C_{55} \gamma_{x z}+C_{56} \gamma_{x y}\right)+\gamma_{x y} \frac{1}{2} C_{66} \gamma_{x y} .
\end{gathered}
$$

Expressions (1) - (3) in system (5) yield $W_{e}=W_{e}(\{\delta\})$. The fulfilled condition $\frac{\partial W_{e}(\{\delta\})}{\partial \delta_{i}}=k_{i j}$, where $i=1, \ldots, 3 n$ and $j=i, \ldots, 3 n$, is applied to determine the coefficients $k_{i j}$ of the top triangular part of the stiffness matrix (a dimension of $3 n \times 3 n$ ) of the threedimensional FE $V_{e}$, which, based on Eq. (4), are written as

$$
\begin{aligned}
& k_{\alpha \beta}=C_{11} A_{\alpha \beta}+C_{15}\left(P_{\alpha \beta}+P_{\beta \alpha}\right)+C_{16}\left(D_{\alpha \beta}+D_{\beta \alpha}\right)+C_{56}\left(F_{\alpha \beta}+F_{\beta \alpha}\right)+ \\
& +C_{55} Q_{\alpha \beta}+C_{66} B_{\alpha \beta}, \\
& k_{\alpha+n, \beta+n}=C_{22} B_{\alpha \beta}+C_{24}\left(F_{\alpha \beta}+F_{\beta \alpha}\right)+C_{46}\left(P_{\alpha \beta}+P_{\beta \alpha}\right)+C_{26}\left(D_{\alpha \beta}+D_{\beta \alpha}\right)+ \\
& +C_{44} Q_{\alpha \beta}+C_{66} A_{\alpha \beta} \text {, } \\
& k_{\alpha+2 n, \beta+2 n}=C_{33} Q_{\alpha \beta}+C_{34}\left(F_{\alpha \beta}+F_{\beta \alpha}\right)+C_{35}\left(P_{\alpha \beta}+P_{\beta \alpha}\right)+C_{45}\left(D_{\alpha \beta}+D_{\beta \alpha}\right)+ \\
& +C_{44} B_{\alpha \beta}+C_{55} A_{\alpha \beta} \text {, } \\
& \alpha=1, \ldots, n, \quad \beta=\alpha, \ldots, n \text {; } \\
& k_{\alpha, \beta+n}=C_{12} D_{\alpha \beta}+C_{14} P_{\alpha \beta}+C_{56} P_{\beta \alpha}+C_{25} F_{\beta \alpha}+C_{26} B_{\alpha \beta}+C_{45} Q_{\alpha \beta}+ \\
& +C_{16} A_{\alpha \beta}+C_{66} D_{\beta \alpha}+C_{46} F_{\alpha \beta}, \\
& k_{\alpha, \beta+2 n}=C_{13} P_{\alpha \beta}+C_{14} D_{\alpha \beta}+C_{15} A_{\alpha \beta}+C_{35} Q_{\alpha \beta}+C_{36} F_{\alpha \beta}+C_{45} F_{\beta \alpha}+ \\
& +C_{46} B_{\alpha \beta}+C_{55} P_{\beta \alpha}+C_{56} D_{\beta \alpha}, \\
& k_{\alpha+n, \beta+2 n}=C_{23} F_{\alpha \beta}+C_{24} B_{\alpha \beta}+C_{25} D_{\beta \alpha}+C_{34} Q_{\alpha \beta}+C_{36} P_{\alpha \beta}+C_{45} P_{\beta \alpha}+ \\
& +C_{46} D_{\alpha \beta}+C_{44} F_{\beta \alpha}+C_{56} A_{\alpha \beta}, \quad \alpha=1, \ldots, n, \quad \beta=1, \ldots, n ; \\
& A_{\alpha \beta}=\int_{V_{e}} \frac{\partial N_{\alpha}}{\partial x} \frac{\partial N_{\beta}}{\partial x} d V, \quad B_{\alpha \beta}=\int_{V_{e}} \frac{\partial N_{\alpha}}{\partial y} \frac{\partial N_{\beta}}{\partial y} d V, \\
& Q_{\alpha \beta}=\int_{V_{e}} \frac{\partial N_{\alpha}}{\partial z} \frac{\partial N_{\beta}}{\partial z} d V, \alpha=1, \ldots, n, \quad \beta=\alpha, \ldots, n \\
& P_{\alpha \beta}=\int_{V_{e}} \frac{\partial N_{\alpha}}{\partial x} \frac{\partial N_{\beta}}{\partial z} d V, \quad D_{\alpha \beta}=\int_{V_{e}} \frac{\partial N_{\alpha}}{\partial x} \frac{\partial N_{\beta}}{\partial y} d V, \\
& F_{\alpha \beta}=\int_{V_{e}} \frac{\partial N_{\alpha}}{\partial y} \frac{\partial N_{\beta}}{\partial z} d V, \alpha=1, \ldots, n, \quad \beta=1, \ldots, n ;
\end{aligned}
$$

where $C_{i j}$ denotes the elastic moduli and $V_{e}$ is the domain of the FE $V_{e}$.

The coefficients of the bottom triangular part of the stiffness matrix of the FE $V_{e}$ are determined from the condition of its symmetry, i.e. $k_{\beta \alpha}=k_{\alpha \beta}$, where $\alpha=1, \ldots, 3 n$ and $\beta=\alpha, \ldots, 3 n$. 
Let there be an arbitrarily shaped homogeneous $\mathrm{FE} S_{e}$ of any order in a plane problem of the elasticity theory, located in a Cartesian coordinate system $O x y$. The stresses $\sigma_{x}$, $\sigma_{y}$, and $\tau_{y z}$, deformations $\varepsilon_{x}, \varepsilon_{y}$, and $\gamma_{y x}$, and displacements $u$ and $v$ of the element $S_{e}$ are related by the Hooke and Cauchy expressions [16]

$$
\begin{gathered}
\{\sigma\}=[D]\{\varepsilon\}, \\
\varepsilon_{x}=\frac{\partial u}{\partial x}, \quad \varepsilon_{y}=\frac{\partial v}{\partial y}, \gamma_{x y}=\frac{\partial u}{\partial y}+\frac{\partial v}{\partial x},
\end{gathered}
$$

where $\{\sigma\}=\left\{\sigma_{x}, \sigma_{y}, \tau_{x y}\right\}^{T}$ and $\{\varepsilon\}=\left\{\varepsilon_{x}, \varepsilon_{y}, \gamma_{x y}\right\}^{T}$ denotes the stress and strain vectors, $u$ and $v$ are the displacement vectors of the element $S_{e},[D]$ is the matrix of the elastic moduli $C_{i j}$ of the FE $S_{e}$, and $C_{i j}=C_{j i}, i, j=1, \ldots, 3$.

Based on the FEM, the approximating displacement functions $u$ and $v$ of the element $S_{e}$ are determined from the expression

$$
\left\{\begin{array}{l}
u \\
v
\end{array}\right\}=[N]\{\delta\}
$$

where $\{\delta\}=\left\{\delta_{1}, \ldots, \delta_{2 n}\right\}^{T}$ is the vector of the nodal unknowns of the FE $S_{e}$, $\delta_{1}, \ldots, \delta_{n}$ denotes the nodal values of the displacements $u$, and $\delta_{n+1}, \ldots, \delta_{2 n}$ denotes the nodal values of the displacements $v$, i.e., the matrix of the shape functions $[N]$ has the form

$$
[N]=\left[\begin{array}{cc}
N_{1}, \ldots, N_{n}, & 0, \ldots, 0 \\
0, \ldots, 0, & N_{1}, \ldots, N_{n}
\end{array}\right],
$$

where $N_{\alpha}$ denotes the shape functions of the FE $S_{e}$ and $\alpha=1, \ldots, n$, where $n$ is the total number of shape functions.

The strain energy $W_{e}$ of the element $S_{e}$, which is used to determine the stiffness matrix of the FE $S_{e}$, can be written in the form

$$
W_{e}=\varepsilon_{x}\left(\frac{1}{2} C_{11} \varepsilon_{x}+C_{12} \varepsilon_{y}+C_{13} \gamma_{y x}\right)+\varepsilon_{y}\left(\frac{1}{2} C_{22} \varepsilon_{y}+C_{23} \gamma_{y x}\right)+\gamma_{x y} \frac{1}{2} C_{33} \gamma_{x y} .
$$

Expressions (8) and (9) in the representation (11) yield $W_{e}=W_{e}(\{\delta\})$. The fulfilled condition $\frac{\partial W_{e}(\{\delta\})}{\partial \delta_{i}}=k_{i j}$, where $i=1, \ldots, 2 n$ and $j=i, \ldots, 2 n$, is used to determine the coefficients $k_{i j}$ of the top triangular part of the stiffness matrix (a dimension of $2 n \times 2 n$ ) of the FE $S_{e}$ in the plane problem of the elasticity theory, which, based on Eq. (14), are written as

$$
\begin{gathered}
k_{\alpha \beta}=C_{11} A_{\alpha \beta}+C_{13}\left(D_{\alpha \beta}+D_{\beta \alpha}\right)+C_{33} B_{\alpha \beta}, \\
k_{\alpha+n, \beta+n}=C_{22} B_{\alpha \beta}+C_{23}\left(D_{\alpha \beta}+D_{\beta \alpha}\right)+C_{33} A_{\alpha \beta}, \alpha=1, \ldots, n, \beta=\alpha, \ldots, n ; \\
k_{\alpha, \beta+n}=C_{12} D_{\alpha \beta}+C_{13} A_{\alpha \beta}+C_{23} B_{\alpha \beta}+C_{33} D_{\beta \alpha}, \alpha, \beta=1, \ldots, n, \\
A_{\alpha \beta}=\int_{S_{e}} \frac{\partial N_{\alpha}}{\partial x} \frac{\partial N_{\beta}}{\partial x} d S, \quad B_{\alpha \beta}=\int_{S_{e}} \frac{\partial N_{\alpha}}{\partial y} \frac{\partial N_{\beta}}{\partial y} d S, \alpha=1, \ldots, n, \beta=\alpha, \ldots, n ;
\end{gathered}
$$




$$
D_{\alpha \beta}=\int_{S_{e}} \frac{\partial N_{\alpha}}{\partial x} \frac{\partial N_{\beta}}{\partial y} d S, \quad \alpha, \beta=1, \ldots, n,
$$

where $C_{i j}$ denotes the elastic moduli and $S_{e}$ is the domain of the FE $S_{e}$.

The coefficients of the bottom triangular part of the stiffness matrix of the element $S_{e}$ are determined from the condition of its symmetry, i.e., $k_{\beta \alpha}=k_{\alpha \beta}$, where $\alpha=1, \ldots, 2 n$ and $\beta=\alpha, \ldots, 2 n$.

\section{Formulations of the Theorems for Isotropic Homogeneous Finite Elements. Construction of $R$ Relations}

Two- and three-dimensional composite constructions of regular structure, which, according to the macroapproach, can be considered as isotropic homogeneous bodies and whose regular cells are cube-shaped (square-shaped) are frequently used in practice. Based on expressions (6), (7), (12), and (13), mutually unambiguous relations are established between the elastic moduli and stiffness matrix coefficients of isotropic homogeneous FE, which are reflected in the following theorems.

Theorem 1. Let Hooke's law and Cauchy expressions be valid for the square-shaped linear-elastic isotropic homogeneous FE $S_{e}$ of the first order in the plane problem of the elasticity theory, located in the Cartesian coordinate system $O x y$. Then parameters $\delta_{1}, \delta_{2}$, $\delta_{3}$, and $\delta_{4}$ can be selected from the vector of the nodal unknowns of the FE $S_{e}$ and the shape function of the FE $S_{e}$ of the form (9), (10), $n=4$, and Eqs. (12) and (13) can be used to construct square matrices $\left[H_{1}\right]$ and $\left[H_{2}\right]$ such that $\left\{K_{1}\right\}=\left[H_{1}\right]\left\{C_{1}\right\}$ and $\left\{K_{2}\right\}=\left[H_{2}\right]\left\{C_{2}\right\}$ or

$$
\left\{C_{1}\right\}=\left[H_{1}\right]^{-1}\left\{K_{1}\right\},\left\{C_{2}\right\}=\left[H_{2}\right]^{-1}\left\{K_{2}\right\},
$$

where $\left\{C_{1}\right\}=\left\{C_{11}, C_{13}, C_{33}\right\}^{T},\left\{C_{2}\right\}=\left\{C_{22}, C_{23}, C_{33}\right\}^{T},\left\{K_{1}\right\}=\left\{k_{11}, k_{12}, k_{22}\right\}^{T}$, $\left\{K_{2}\right\}=\left\{k_{33}, k_{34}, k_{44}\right\}^{T} ;\left[H_{1}\right]^{-1}$ and $\left[H_{2}\right]^{-1}$ are the matrices inverse to $\left[H_{1}\right]$ and $\left[H_{2}\right]$; $k_{i j}$ denotes the coefficients of the stiffness matrix of the FE $S_{e}$, which satisfies a system of relations of the FEM of the form

$$
\left[\begin{array}{l}
k_{11}, k_{12}, k_{13}, k_{14} \\
k_{21}, k_{22}, k_{23}, k_{24} \\
k_{31}, k_{32}, k_{33}, k_{34} \\
k_{41}, k_{42}, k_{43}, k_{44}
\end{array}\right]\left\{\begin{array}{l}
\delta_{1} \\
\delta_{2} \\
\delta_{3} \\
\delta_{4}
\end{array}\right\}=\left\{\begin{array}{l}
R_{1} \\
R_{2} \\
R_{3} \\
R_{4}
\end{array}\right\},
$$

where $R_{i}$ denotes the nodal forces of the FE, $i=1, \ldots, 4$, corresponding to the nodal displacements $\delta_{1}, \delta_{2}, \delta_{3}$, and $\delta_{4}$.

In other words, the stiffness matrix of the square-shaped linear-elastic isotropic homogeneous $\mathrm{FE}$ of the first order in the plane problem of the elasticity theory always contains six coefficients such that can be used to determine five elastic moduli $C_{11}, C_{13}$, $C_{22}, C_{23}$, and $C_{33}$ of this FE.

The elastic modulus $C_{12}$ is determined as follows. Expressions (12) and (13) are used to construct a matrix relation

$$
\left\{K_{3}\right\}=\left[H_{3}\right]\left\{C_{3}\right\},
$$


where $\left\{C_{3}\right\}=\left\{C_{12}, C_{13}, C_{23}, C_{33}\right\}^{T}, \quad\left\{K_{3}\right\}=\left\{k_{13}, k_{14}, k_{23}, k_{24}\right\}^{T}, \quad$ and $\quad\left[H_{3}\right] \quad$ is the $4 \times 4$ matrix.

Let $h_{i j}^{3}$ be denoted by the coefficients of the matrix $\left[H_{3}\right], i, j=1, \ldots, 4$. For example, let $h_{i 1}^{3} \neq 0 \quad(i=1, \ldots, 4)$. Then, according to Eq. (16), the elastic modulus $C_{12}$ is expressed via $C_{13}, C_{23}$, and $C_{33}$ according to the equation

$$
C_{12}=\left(k_{i}^{*}-C_{13} h_{i 2}^{3}-C_{23} h_{i 3}^{3}-C_{33} h_{i 4}^{3}\right) / h_{i 1}^{3},
$$

where $i=1, \ldots, 4, k_{1}^{*}=k_{13}, k_{2}^{*}=k_{14}, k_{3}^{*}=k_{23}$, and $k_{4}^{*}=k_{24}$.

Expressions (14) and (17) represent $R$ relations. These relations are used to determine apparent elastic moduli for two-dimensional composite bodies with square-shaped regular cells. The procedure of calculating the apparent elastic moduli for such two-dimensional composite bodies is described in detail in $[17,18]$.

Note 1. It is noteworthy that constructing the relations (14) and (17) (i.e., $R$ relations) only requires determining the matrices $\left[H_{1}\right],\left[H_{2}\right]$, and $\left[H_{3}\right]$, which can be formed using the shape functions of the FE $S_{e}$ of the form (9), (10), $n=4$, and expressions (12) and (13). The coefficients of the matrices $\left[H_{1}\right],\left[H_{2}\right]$, and $\left[H_{3}\right]$ are determined using expressions (13).

Theorem 2. Let Hooke's law and Cauchy expressions be valid for the cube-shaped linear-elastic isotropic homogeneous $\mathrm{FE} V_{e}$ of the first order in the three-dimensional problem of the elasticity theory, located in the Cartesian coordinate system Oxyz. Then the nodal parameters $\delta_{1}, \delta_{2}, \delta_{3}, \delta_{4}, \delta_{5}, \delta_{6}, \delta_{7}, \delta_{8}$, and $\delta_{9}$ can be selected from the vector of the nodal unknowns of the FE $V_{e}$, and the shape functions of the FE of the form (3), (4), $n=8$, and expressions (6) and (7) can be used to construct such square matrices $\left[H_{1}\right],\left[H_{2}\right]$, and $\left[H_{3}\right]$, for which the following equalities are valid: $\left\{K_{1}\right\}=\left[H_{1}\right]\left\{C_{1}\right\}$, $\left\{K_{2}\right\}=\left[H_{2}\right]\left\{C_{2}\right\}$, and $\left\{K_{3}\right\}=\left[H_{3}\right]\left\{C_{3}\right\}$ or

$$
\left\{C_{1}\right\}=\left[H_{1}\right]^{-1}\left\{K_{1}\right\},\left\{C_{2}\right\}=\left[H_{2}\right]^{-1}\left\{K_{2}\right\}, \quad\left\{C_{3}\right\}=\left[H_{3}\right]^{-1}\left\{K_{3}\right\},
$$

where $\left\{C_{1}\right\}=\left\{C_{11}, C_{15}, C_{16}, C_{55}, C_{56}, C_{66}\right\}^{T},\left\{C_{2}\right\}=\left\{C_{22}, C_{24}, C_{26}, C_{44}, C_{46}, C_{66}\right\}^{T}$, $\left\{C_{3}\right\}=\left\{C_{33}, C_{34}, C_{35}, C_{44}, C_{45}, C_{55}\right\}^{T},\left\{K_{1}\right\}=\left\{k_{11}, k_{12}, k_{13}, k_{22}, k_{23}, k_{33}\right\}^{T}$, $\left\{K_{2}\right\}=\left\{k_{44}, k_{45}, k_{46}, k_{55}, k_{56}, k_{66}\right\}^{T},\left\{K_{3}\right\}=\left\{k_{77}, k_{78}, k_{79}, k_{88}, k_{89}, k_{99}\right\}^{T},\left[H_{1}\right]^{-1}$, $\left[H_{2}\right]^{-1}$ and $\left[H_{3}\right]^{-1}$ are the matrices inverse to $\left[H_{1}\right],\left[H_{2}\right]$, and $\left[H_{3}\right]$, and $k_{i j}$ denotes the stiffness matrix coefficients of the FE $V_{e}$, which satisfy the system of FEM expressions of the form

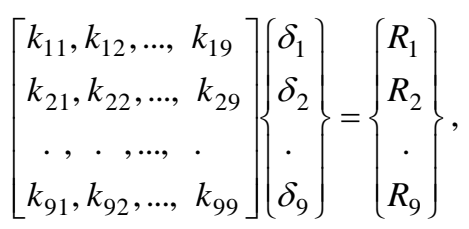

where $R_{i}$ denotes the nodal forces of the FE $V_{e}$, in which the FEM parameters $\delta_{i}$, $i=1, \ldots, 9$ are determined.

In other words, the stiffness matrix of the cube-shaped linear-elastic isotropic homogeneous $\mathrm{FE}$ of the first order in the three-dimensional problem of the elasticity theory always contains 18 
coefficients such that can be used to determine 15 elastic moduli of this FE: $C_{11}, C_{15}, C_{16}$, $C_{22}, C_{24}, C_{26}, C_{33}, C_{34}, C_{35}, C_{44}, C_{45}, C_{46}, C_{55}, C_{56}$, and $C_{66}$.

The remaining six elastic moduli of the $\mathrm{FE} V_{e}$ are unambiguously determined via the elastic moduli (18) as follows. Equations (6) and (7) are used to construct expressions

$$
\left\{K_{4}\right\}=\left[H_{4}\right]\left\{C_{4}\right\}+\left[G_{4}\right]\left\{C_{4}^{*}\right\}, \quad\left\{K_{5}\right\}=\left[H_{5}\right]\left\{C_{5}\right\}+\left[G_{5}\right]\left\{C_{5}^{*}\right\},
$$

where $\left\{K_{4}\right\}=\left\{k_{14}, k_{15}, k_{16}\right\}^{T} ;\left\{K_{5}\right\}=\left\{k_{48}, k_{49}, k_{57}\right\}^{T} ;\left\{C_{4}\right\}=\left\{C_{12}, C_{14}, C_{25}\right\}^{T}$;

$\left\{C_{5}\right\}=\left\{C_{23}, C_{25}, C_{36}\right\}^{T} ;\left\{C_{4}^{*}\right\}=\left\{C_{16}, C_{26}, C_{45}, C_{46}, C_{56}, C_{66}\right\}^{T}$;

$\left\{C_{5}^{*}\right\}=\left\{C_{24}, C_{34}, C_{44}, C_{45}, C_{46}, C_{56}\right\}^{T} ;\left[H_{4}\right]$ and $\left[H_{5}\right]$ denote $3 \times 3$ matrices, and $\left[G_{4}\right]$ and $\left[G_{5}\right]$ are $3 \times 6$ matrices.

The calculations show that $\operatorname{det}\left[H_{4}\right] \neq 0$ and $\operatorname{det}\left[H_{5}\right] \neq 0$. Then expressions (19) yield vectors $\left\{C_{4}\right\}$ and $\left\{C_{5}\right\}$ using equations

$$
\begin{gathered}
\left\{C_{4}\right\}=\left[H_{4}\right]^{-1}\left\{K_{4}\right\}-\left[H_{4}\right]^{-1}\left[G_{4}\right]\left\{C_{4}^{*}\right\}, \\
\left\{C_{5}\right\}=\left[H_{5}\right]^{-1}\left\{K_{5}\right\}-\left[H_{5}\right]^{-1}\left[G_{5}\right]\left\{C_{5}^{*}\right\},
\end{gathered}
$$

where $\left[H_{4}\right]^{-1}$ and $\left[H_{5}\right]^{-1}$ are the matrices inverse to $\left[H_{4}\right]$ and $\left[H_{5}\right]$.

Note that the elastic moduli vectors $\left\{C_{4}^{*}\right\}$ and $\left\{C_{5}^{*}\right\}$ in expressions (20) are known (see Eqs. (18)). Thus, only $C_{13}$ is left to be determined. For that purpose, Eqs. (6) and (7) are used to construct an expression

$$
\left\{K_{6}\right\}=\left[H_{6}\right]\left\{C_{6}\right\},
$$

where $\left[H_{6}\right]$ denotes a $9 \times 9$ matrix, $\left\{K_{6}\right\}=\left\{k_{17}, k_{18}, k_{19}, k_{27}, k_{28}, k_{29}, k_{37}, k_{38}, k_{39}\right\}^{T}$, and $\left\{C_{6}\right\}=\left\{C_{13}, C_{14}, C_{15}, C_{35}, C_{36}, C_{45}, C_{46}, C_{55}, C_{56}\right\}^{T}$.

Let $h_{i j}^{6}$ denote the coefficients of the matrix $\left[H_{6}\right], i, j=1, \ldots 9$. For example, let $h_{i 1}^{6} \neq 0$. Then, according to Eq. (21), the elastic modulus $C_{13}$ is calculated from

$$
\begin{gathered}
C_{13}=\left(k_{i}-C_{14} h_{i 2}^{6}-C_{15} h_{i 3}^{6}-C_{35} h_{i 4}^{6}-C_{36} h_{i 5}^{6}-C_{45} h_{i 6}^{6}-\right. \\
\left.-C_{46} h_{i 7}^{6}-C_{55} h_{i 8}^{6}-C_{56} h_{i 9}^{6}\right) / h_{i 1}^{6},
\end{gathered}
$$

where $k_{i}^{*}$ - denotes the parameter of the vector $\left\{K_{6}\right\}, i=1, \ldots, 9, k_{1}^{*}=k_{17}, k_{2}^{*}=k_{18}$, $k_{3}^{*}=k_{19}, \ldots, k_{9}^{*}=k_{39}$.

Expressions (18), (20), and (22) represent the $R$ relations. These relations are used to determine the apparent elastic moduli for three-dimensional composite bodies with squareshaped regular cells. How apparent moduli for these three-dimensional composite bodies can be determined is described in detail in [19].

Note 2. It is noteworthy that constructing relations (18), (20), and (22) (i.e., $R$ relations) only requires determining the matrices $\left[H_{1}\right],\left[H_{2}\right],\left[H_{3}\right],\left[H_{4}\right],\left[H_{5}\right],\left[H_{6}\right],\left[G_{4}\right]$, and $\left[G_{5}\right]$, which are formed using the shape functions of the FE $V_{e}$ of the form (3), (4), $n=8$, and expressions (6) and (7). The coefficients of the matrices $\left[H_{1}\right], \ldots,\left[G_{5}\right]$ are determined using Eqs. (7).

Note 3. As shown by the calculations, theorems 1 and 2 are also valid for the high-order square- and cube-shaped isotropic homogeneous FE $S_{e}$ and $V_{e}$. However, it is reasonable 
to use the first-order FE to determine the apparent elastic moduli as the increase in the order of the FE $S_{e}$ and $V_{e}$ makes the volume of computations larger.

\section{Main provisions for determining apparent elastic moduli}

Main provisions for determining apparent elastic moduli are considered on the example of a two-dimensional composite body $S_{p}$ of regular structure, which is subjected to a plane stress state and located in the Cartesian coordinate system $O x y$. The presence of ideal relations between the components of the composite body $S_{p}$ is assumed. According to the macroapproach, the body $S_{p}$ can be regarded as an isotropic homogeneous body. A representative FE (RFE) comprised of a finite number of square regular cells of inhomogeneous structure of the body $S_{p}$ is used a representative volume of the composite body $S_{p}$. Let the RFE be square-shaped with side $a$. The domain of the RFE is represented by a basic partition $R_{h}$ consisting of a square-shaped FE $S_{i}^{h}$ of the first order with side $h, i=1, \ldots, N$, where $N$ is the total number of the FE $S_{i}^{h}$. The basic partition accounts for the inhomogeneous structure of the RFE and generates a square fine grid $S_{h}$ with cell $h$. The functional $W_{p}$ of the total potential energy of the RFE is written as

$$
W_{p}=\sum_{i=1}^{N}\left(0,5\left\{q_{i}\right\}^{T}\left[K_{i}\right]\left\{q_{i}\right\}-\left\{q_{i}\right\}^{T}\left\{R_{i}\right\}\right)
$$

where $\left[K_{i}\right]$ - denotes the stiffness matrix of the element $S_{i}^{h}$, and $\left\{q_{i}\right\},\left\{R_{i}\right\}$ are the vectors of the nodal unknowns and nodal forces of the element $S_{i}^{h}$.

A coarse grid $S_{H}$ is determined in the fine grid $S_{h}$, and the nodes of the coarse grid are the apexes of the RFE, i.e., the grid $S_{H}$ has four nodes. In the coarse grid $S_{H}$, the FEM is used to determine the approximating displacement functions $u$ and $v$ of the form (9), (10), $n=4$. The resulting representations $u=\sum_{i=1}^{4} N_{i} \delta_{i}^{H} \quad$ and $\quad v=\sum_{i=1}^{4} N_{i} \delta_{i+4}^{H} \quad$ (where $\delta_{i}^{H}$ denotes the nodal unknowns of the coarse grid $\left.S_{H}, i=8\right)$ are used to express the vector $\left\{q_{i}\right\}$ of the nodal unknowns of the $\mathrm{FE} S_{i}^{h}$ via the vector $\left\{q_{H}\right\}$ of nodal unknowns of the coarse grid $S_{H}$ of the RFE. Thus, the following equation is obtained:

$$
\left\{q_{i}\right\}=\left[A_{i}\right]\left\{q_{H}\right\},
$$

where $\left[A_{i}\right]$ denotes an $8 \times 8$ square matrix. Equation (24) is substituted into Eq. (23), and condition $\partial W_{p}\left(\left\{q_{H}\right\}\right) / \partial\left\{q_{H}\right\}=0$ yields

$$
\left[K_{p}\right]=\sum_{i=1}^{N}\left[A_{i}\right]^{T}\left[K_{i}\right]\left[A_{i}\right],\left\{F_{p}\right\}=\sum_{i=1}^{N}\left[A_{i}\right]^{T}\left\{R_{i}\right\}
$$

where $\left[K_{p}\right],\left\{F_{p}\right\}$ are the stiffness matrix and the vector of nodal forces of the RFE.

It is noteworthy that a first-order two-grid FE $(2 \mathrm{gFE})$ is constructed on the domain of the RFE [6-8], i.e., $\left[K_{p}\right]$ is the stiffness matrix, $\left\{F_{p}\right\}$ is the vector of nodal forces, 
and $R_{h}$ is the basic partition of the $2 \mathrm{gFE}$. The latter, i.e., RFE is known to be arbitrarily small, so it can arbitrarily accurately account for the inhomogeneous (microinhomoge- neous) structure of the RFE. One should pay attention to the provisions on the basis of which the apparent elastic moduli are determined. According to the macroapproach, the RFE is regarded as a square-shaped isotropic homogeneous FE of the first order (i.e., square-shaped $2 \mathrm{gFE}$ of the first order) with apparent elastic moduli $C_{i j}^{p}$. Expressions (23)-(25) are used to determine the coefficients $k_{i j}^{p}$ of the stiffness matrix $\left[K_{p}\right]$ of the RFE, $i, j=1, \ldots 8$. Then, according to Theorem 1 , the elastic moduli $C_{i j}^{p}$ and six coefficients $k_{i j}^{p}$ of the stiffness matrix $\left[K_{p}\right]$ of the RFE are related by $R$ relations of the form (14), (17). Consequently, the apparent elastic moduli $C_{i j}^{p}$ for the RFE (i.e., composite body $S_{p}$ ) are calculated using Eqs. (14) and (17), in which the elastic moduli $C_{i j}$ and coefficients $k_{i j}$ are respectively replaced by the apparent elastic moduli $C_{i j}^{p}$ and coefficients $k_{i j}^{p}$, i.e.,

$$
\begin{aligned}
\left\{C_{1}^{p}\right\} & =\left[H_{1}\right]^{-1}\left\{K_{1}^{p}\right\}, \quad\left\{C_{2}\right\}=\left[H_{2}\right]^{-1}\left\{K_{2}\right\}, \\
C_{12}^{p} & =\left(k_{i}^{*}-C_{13}^{p} h_{i 2}^{3}-C_{23}^{p} h_{i 3}^{3}-C_{33}^{p} h_{i 4}^{3}\right) / h_{i 1}^{3},
\end{aligned}
$$

where $\quad\left\{K_{1}^{p}\right\}=\left\{k_{11}^{p}, k_{12}^{p}, k_{22}^{p}\right\}^{T}, \quad\left\{K_{2}^{p}\right\}=\left\{k_{33}^{p}, k_{34}^{p}, k_{44}^{p}\right\}^{T} ; \quad\left\{C_{1}^{p}\right\}=\left\{C_{11}^{p}, C_{13}^{p}, C_{33}^{p}\right\}^{T}$, $\left\{C_{2}^{p}\right\}=\left\{C_{22}^{p}, C_{23}^{p}, C_{33}^{p}\right\}^{T}, i=1,2,3, k_{1}^{*}=k_{13}^{p}, k_{2}^{*}=k_{14}^{p}, k_{3}^{*}=k_{23}^{p}, h_{i 1}^{3} \neq 0$.

The procedures of constructing the matrices $\left[H_{1}\right],\left[H_{2}\right]$, and $\left[H_{3}\right]$ are briefly described in Note 1. It is well-known [14] that the elastic moduli $C_{i j}, C_{j i}=C_{i j}, i=1, . ., 3$, $j=i, \ldots, 3$, of isotropic homogeneous two-dimensional bodies subjected to a plane stress state satisfy the conditions

$$
C_{11}=C_{22}, C_{13}=C_{23}=0 .
$$

The calculations show that, as $a$ increases, $C_{i j}^{p}$ tend to the limiting values of $C_{i j}^{0}$, i.e., $C_{i j}^{p} \rightarrow C_{i j}^{0}$ as $a \rightarrow a_{0}, i, j=1, \ldots, 3$. Then

$$
\text { for } a \geq a_{0}: C_{i j}^{p}=C_{i j}^{0}, \quad i, j=1,2,3 .
$$

According to the calculations, for the two-dimensional composite bodies $S_{p}$ of regular structure, with square-shaped regular cells, there is such a small value $\varepsilon \geq 0$ that

$$
\left|C_{11}^{0}-C_{22}^{0}\right| /\left|C_{22}^{0}\right| \leq \varepsilon,\left|C_{13}^{0}\right|,\left|C_{23}^{0}\right| \leq \varepsilon .
$$

In view of Eqs. (27), (28), and (29), the RFE (i.e., two-dimensional composite body $S_{p}$ ) with a characteristic dimension $a \geq a_{0}$ can be regarded as an isotropic homogeneous body. The calculations confirm that the apparent elastic moduli for a specified class of composite bodies of regular structure satisfy the conditions of isotropic homogeneous bodies. Determining the apparent elastic moduli is described in detail in [17] and [18] for two-dimensional composite bodies of regular structure and in [19] for three-dimensional composite bodies. 


\section{Conclusion}

In this paper the procedure is proposed for determining apparent elastic moduli of two- or three-dimensional composite bodies of regular structure with an arbitrary filling ratio, which has a matrix formulation, and is implemented on the basis of finite element method algorithms. It is shown that apparent elastic moduli determined for a certain class of composite bodies of regular structure satisfy the conditions of isotropic homogeneous bodies.

\section{References}

1. D.H. Norrie, G. de Vries. An Introduction to Finite Element Analysis (Academic Press, New York; San Francisco, London, 1978)

2. O.C. Zienkiewicz. The Finite Element Method in Engineering Science (McGraw-Hill, London, 1971)

3. K.-J. Bathe, E.L. Wilson. Numerical Methods in Finite Element Analysis (Prentice Hall, Inc., 1976)

4. M. Sekulovich. Finite Element Method (Stroiizdat, Moscow, 1993) [in Russian]

5. T. Fuji and J. Jako. Mechanics of the Fracture of Composite Materials (Mir, Moscow, 1982)

6. A.D. Matveev. IOP Conf. Ser.: Mater. Sci. Eng. 158, 1, 012067, 1-9 (2016)

7. A.D. Matveev, Vestnik KrasGAU, 12, 93-100 (2016)

8. A.D. Matveev. Uchen. Zap. Kazan. Univ. Seriya: Fiz.-Matem. Nauki 158 (4), 530-543 (2016)

9. N.A. Alfutov, P. A. Zinov'ev, and B.G. Popov. Calculation of Multilayer Plates and Shells Made of Composite Materials (Mashinostroenie, Moscow, 2008)

10. S.K. Golushko, Yu.V. Nemirovskii. Direct and Inverse Problems of Mechanics of Elastic Composite Plates and Shells of Revolution (Fizmatlit, Moscow, 2008)

11. A. Ahmed A, S. Kapuria, Composite Structures, 158, 112-127 (2016)

12. M. Cinefra, E. Carrera, Int. J. Num. Meth. Eng. 93, 2, 160-182 (2013)

13. E. Carrera, A. Pagani, S. Valvano. Composites Part B: Engineering 111, $294-314$ (2017)

14. M.Y. Yasin, S. Kapuria. Composite Structures 98, 202-214 (2013)

15. M.F. Caliri, A.J.M. Ferreira, V. Tita, Composite Structures 156, 63-77 (2016)

16. V.I. Samul', Fundamentals of the Theory of Elasticity and Plasticity (Vyssh. Shk., Moscow, 1970)

17. A.D. Matveev. Vestnik KrasGAU, 12, 212-222 (2006)

18. A.D. Matveev, Proceedings of the XXI All-Russia Conference "Numerical Methods for Solving the Problems of Elasticity and Plasticity". Parallel, Novosibirsk, 158-167 (2009)

19. A.D. Matveev, Vestnik KrasGAU, 5, 34-37 (2008) 